\title{
OTOLOGY
}

\section{Otologic manifestations of Susac syndrome}

\author{
Manifestazioni otologiche della sindrome di Susac \\ V.A. PATEL ${ }^{1}$, M. DUNKLEBARGER ${ }^{2}$, T.T. ZACHARIA ${ }^{3}$, H. ISILDAK ${ }^{1}$ \\ ${ }^{1}$ Department of Surgery, Division of Otolaryngology - Head and Neck Surgery, The Pennsylvania State University, \\ College of Medicine, Hershey, PA, USA; ${ }^{2}$ The Pennsylvania State University, College of Medicine, Hershey, PA, \\ USA; ${ }^{3}$ Department of Radiology, The Pennsylvania State University, College of Medicine, Hershey, PA, USA
}

\section{SUMMARY}

\begin{abstract}
Susac syndrome, a rare autoimmune disorder first described as a classic triad (encephalopathy, branch retinal artery occlusion, and sensorineural hearing loss) in 1979 by renowned physician John O. Susac, has been an advancing area of clinical interest and scientific research over the last several decades. This comprehensive review aims to succinctly highlight the breadth and detail of this enigmatic disease, with a primary focus on otologic manifestations. Topics discussed include epidemiology, pathophysiology, clinical manifestations, differential diagnoses, classification schema, laboratory investigations, characteristic audiometric findings, high-yield radiographic imaging, temporal bone histopathology, treatment strategies and overall prognosis.
\end{abstract}

KEY WORDS: Otology • Sensorineural hearing loss $\bullet$ Temporal bone histopathology $\bullet$ Susac syndrome $\bullet$ Update

\section{RIASSUNTO}

La sindrome di Susac, una rara malattia descritta nel 1979 dal celebre medico John O. Susac con la classica triade di encefalopatia, occlusione dell'arteria retinica ed ipoacusia neurosensoriale, ha rappresentato negli ultimi decenni un tema di notevole interesse clinico e di ricerca scientifica. La presente revisione ha lo scopo di evidenziare i dettagli e le dimensioni di questa enigmatica patologia, focalizzandosi principalmente sulle manifestazioni otologiche. Le tematiche discusse includono epidemiologia, fisiopatologia, manifestazioni cliniche, diagnostica differenziale, classificazione, indagini di laboratorio, caratteristiche audiometriche, imaging, anatomopatologia dell'osso temporale, strategie terapeutiche e prognosi.

PAROLE CHIAVE: Otologia $\bullet$ Perdita dell'udito neurosensoriale $\bullet$ Istopatologia dell'osso temporale $\bullet$ Sindrome di Susac $\bullet$ Aggiornamento

Cite this article as: Acta Otorhinolaryngol Ital 2018;38:544-553. https://doi.org/10.14639/0392-100X-2166

(C) Società Italiana di Otorinolaringoiatria e Chirurgia Cervico-Facciale

\section{Introduction}

Susac Syndrome (SS) is a unique clinical condition first described in 1979 by Dr. Susac, who identified two young women with the clinical triad of subacute multifocal encephalopathy, visual changes and hearing loss secondary to systemic microangiopathy of the cerebrum, retina and cochlea ${ }^{1}$. While the underlying aetiology of this disease remains unknown, it is widely believed the observed clinical manifestations are the result of an autoimmune endotheliopathy of the cerebral, retinal and cochlear microvasculature $^{23}$. This hypothesis has been further purported by both ex vivo histopathologic specimens of the cerebrum and temporal bone as well as in vivo findings of the retina via fluorescein angiography ${ }^{4-9}$. Despite significant advances in the understanding of this disorder, the presentation of SS remains highly variable with respect to disease onset, course, duration and prognosis. Similarly, data enumeration on patterns and ramifications of SS are also limited given the small number of cases presently available in the published literature worldwide ${ }^{10}$. The heterogeneity of presenting symptomology associated with this condition, along with its similarities to other more ubiquitous disease states indeed makes SS a challenging undertaking to astutely diagnose. This is particularly true of the otologic manifestations of SS, where the observed sensorineural hearing loss is often irreversible and progressive in nature, despite early and aggressive therapies. This comprehensive review aims to concisely highlight the current understanding of this enigmatic condition with a distinct emphasis on otologic features pertinent to the field of otolaryngology. To briefly summarise, this study was designed and performed using the Preferred Reporting Items for Systematic Reviews and Meta-Analyses 
(PRISMA) Protocol. Independent searches of the PubMed MEDLINE database were performed on February 12, 2018, by the first and second authors to identify studies which specifically described the otologic manifestations of SS using the Boolean method and relevant search term combinations. English and full-length original articles were employed as inclusion criteria to assess for data extraction eligibility. Exclusion criteria include absent full-text articles and non-English articles, resulting in the omission of 33 and 14 articles, respectively. The search strategy is summarised as follows: 250 articles retrieved, 203 full-length titles and abstracts screened, and 13 studies included in final study data synthesis (i.e. 12 case reports and 1 original article). The abstracted clinical information was supplemented with targeted searches to address specific needs identified in writing this comprehensive review.

\section{Discussion}

\section{Epidemiology}

SS is a rare autoimmune disorder with a slight femaleto-male preponderance of 3:1 and predilection in those of Caucasian ancestry ${ }^{11}$. Most reports of SS are derived from the North American and European continents, with the first clinical ramifications of SS noted between 1640 years, with an extended age range of 7-72 years ${ }^{12}$. As SS remains both underdiagnosed and misdiagnosed, this results in an unknown true incidence and prevalence. Currently, there are a total of 304 documented cases of SS worldwide, with a higher reported incidence during the spring and summer months ${ }^{10}{ }^{13}$.

\section{Pathophysiology and clinical manifestations}

Although the exact aetiology of SS remains elusive, histopathological findings support an autoimmune endotheliopathy of cerebral, retinal and cochlear microvasculature (Fig. 1). Visualised changes include an affinity for small $(<100 \mathrm{~mm}$ ) precapillary arterioles with endothelial cell necrosis, basement membrane thickening, inflammatory cell infiltration and complement deposition $(\mathrm{C} 4 \mathrm{~d}$ and $\mathrm{C} 3 \mathrm{~d}$ ). Following this injury, diffuse microvascular changes ultimately result in microischaemia and infarction leading to the observed clinical manifestations of encephalopathy, visual changes, and sensorineural hearing loss ${ }^{14}$. Nevertheless, SS is a considerable challenge to diagnose as it is very uncommon for patients to initially present with all features of the classically described triad (Table I) ${ }^{7}$. There are two broad categories of SS; the first is a primarily encephalopathic presentation with either concurrent or eventual manifestation of visual dis-

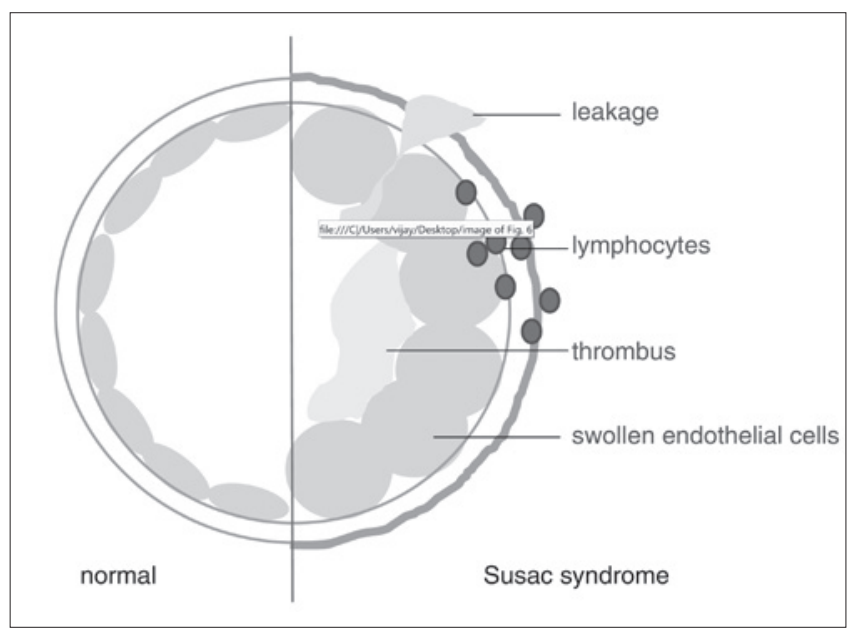

Fig. 1. Proposed model of pathogenesis for Susac syndrome. Used with permission from E. Bernd Ringelstein. The endothelial cells and basement membrane are oedematous, with loss of vessel wall integrity. As a result, there is subsequent lymphocytic infiltration and thrombotic material accumulation within the vessel lumen. This ultimately results in occlusion of the affected microvasculature (from Kleffner et al., $2012^{2}$, mod.).

Table I. Clinical presentation in Susac syndrome.

\begin{tabular}{lc} 
System & Prevalence \\
Brain (corpus callosum) & $88 \%(100 \%)$ \\
Retina & $46 \%$ \\
Cochlea & $52 \%$ \\
All three structures & $20 \%$ \\
\hline
\end{tabular}

Organ involvement at clinical presentation of Susac syndrome (from García-Carrasco et al., $2011^{3}$, mod.).

turbances and sensorineural hearing loss and the second is a recurrent course of branched retinal artery occlusion and hearing loss, which may be accompanied with encephalopathy months to years later ${ }^{15}$.

\section{a. Nervous system}

Encephalopathic features typically follow a subacute or acute course and are often preceded by or concurrent with a migrainous headache, likely secondary to leptomeningeal involvement ${ }^{10}$. Encephalopathic signs may be the initial presentation of SS or secondary to retinal or vestibulocochlear pathology. Encephalopathy often manifests with the abrupt onset of multifocal neurologic deficits and/or neuropsychiatric disturbances. Symptoms may be dominated by confusion, memory and cognitive disturbances or by acute and profound changes in mood and personality. Focal findings such as seizures, pyramidal signs, dysarthria and hyperreflexia may also be present ${ }^{16}$. While generally reversible, some initial deficits may be permanent depending on the location and extent of the involved cerebral vasculopathy. In the original descriptions by Susac and colleagues, multifocal microinfarctions of the cerebral grey and white 
matter were noted with associated necrosis and gliosis ${ }^{1}$. Subsequent developments via brain biopsy highlighted characteristic findings of an autoimmune vasculopathy primarily affecting the precapillary arteriolar endothelium in both the cortex and leptomeninges. Visualised changes also include subacute microinfarctions, perivascular oedema, chronic inflammation and gliosis/reactive astrocytosis ${ }^{45}$. The absence of fibrinoid necrosis, necrotising vasculitis, or demyelination assists in the pathologic distinction of SS from other systemic vasculidities or autoimmune demyelinating conditions ${ }^{67}$.

\section{b. Visual system}

Visual disturbances related to branched retinal artery occlusion are heterogeneous in nature and depend on the location and extent of diseased vessels within the retina. Those with small, peripheral arteriolar occlusions may be asymptomatic or have subtle visual changes, with a normal funduscopic exam ${ }^{17}$. Conversely, those who suffer from more extensive lesions of the retina and macula may experience profound or complete vison loss. Patients may also present with additional ocular complaints of blurred vision, scintillating scotoma, or photopsia ${ }^{18}$. Fluorescein angiography assists in visualising the extent of the occlusive retinal microangiopathy and illustrates characteristic ocular findings such as arteriolar narrowing, arteriolar wall hyperfluorescence, and segmental non-perfusion ${ }^{19}$. Additionally, yellow-white arterial wall plaques (Gass plaques) located at the midarteriolar segments may be seen, proposed to be the result of arteriolar wall damage and subsequent extravasation of blood and lipids forming atheromatous plaques ${ }^{20}$. Their location at the midarteriolar segments is indicative of endothelial integrity loss, rather than an end-artery embolic pathology. These plaques may be transient throughout the clinical progression of this condition; however, their presence is confirmatory of SS 9 .

c. Auditory and vestibular systems

Sensorineural hearing loss is a prominent feature of SS with highly variable characteristics ${ }^{4721-24}$. Hearing loss is often precipitous in onset and typically rapidly progressive, sometimes resulting in complete deafness in either one or both ears. In some instances, hearing loss may abruptly occur in one ear, followed by loss in the contralateral ear days to weeks later, resulting in the so-called "bang-bang" type of hearing loss ${ }^{15}$. Due to the commonly detected pattern of low and middle-frequency sensorineural hearing loss, microinfarctions of end arterioles and damage to the apical cochlea are thought to be responsible in the setting of the observed endotheliopathy ${ }^{61926}$. This pattern of damage is further supported by the clinical observation of sensorineural hearing loss with maintained acoustic reflexes, rather than localised damage to the vestibulocochlear nerve, which suggests cochlear disease as the underlying aetiology ${ }^{24}$. Simultaneous vestibular dysfunction is also common in the early phase of SS; patients can present with severe tinnitus accompanying their abrupt sensorineural hearing loss, which often leads to the misdiagnosis of Ménière's disease ${ }^{27}$. The presence of vestibular dysfunction may indicate lesions in the vestibular apparatus itself or may be the result of central microinfarctions within the cerebellum or brainstem ${ }^{7}$. This is in stark contrast to other autoimmune inner ear diseases such as Cogan syndrome, where the pattern of damage demonstrates extensive endolymphatic hydrops, saccular collapse following intense dilation, fibrotic changes in the perilymphatic space of the posterior semicircular canal, and absorption of enchondral bone with no pathological changes within the inner ear vasculature ${ }^{28}$. Additional symptoms which may be present in SS include gait ataxia, aural pressure, and prominent jerky nystagmus, which is hypothesised to be due to infarction within the membranous labyrinth ${ }^{12}$. In SS patients with prominent encephalopathic features, it may be difficult to assess the presence or extent of vestibulocochlear dysfunction, in which case brainstem auditory evoked potential testing may be necessary ${ }^{18}$.

\section{d. Integumentary and musculoskeletal systems}

Historically, patients have described both myalgias as well as skin changes during the early phase of $\mathrm{SS}^{67}$. Turc and colleagues noted skin lesions consistent with livedo reticularis in a patient with SS; skin biopsies performed demonstrated similar pathologic findings in other affected systems with evidence of microvascular thrombi in dermal arterioles, minimal perivascular lymphocytic infiltration and endothelial cell swelling ${ }^{29}$. Similarly, Petty and colleagues reported subclinical microangiopathy involving muscular arterioles in 5 biopsy specimens with SS, further supporting the notion of a systemic endotheliopathy, unifying features of this condition ${ }^{7}$.

\section{Differential diagnoses and classification schema}

SS is an orphan disease which must be considered in the differential diagnosis of a broad variety of medical disorders ${ }^{30}$. Given the potential for diversely presenting symptomatology, SS remains a pertinent diagnosis chiefly in the realm of neurologic, ophthalmologic, rheumatologic and otolaryngologic practitioners. A concise yet non-exhaustive list which outlines conditions that present with similar clinical characteristics is outlined in Table II. A classification system described by Vishnevskia-Dai and colleagues can also be applied to assist clinicians in the early diagnosis of this enigmatic condition. This schema 
was designed in part using a combination of clinical information derived from the medical literature (case reports, reviews and meta-analysis) as well as the author's case series of 10 patients, with a focus on clinical presentation and diagnostic imaging findings (Table III) ${ }^{31}$. Although novel in applicability, studies have suggested that early diagnosis may lead to better prognosis in young patients, prompting a high level of suspicion and early intervention in patients with $\mathrm{SS}^{30}$.

\section{Diagnostic workup}

\section{a. Audiometry}

Audiological evaluation in the form of pure tone audiom-

Table II. Differential diagnosis of Susac syndrome.

\begin{tabular}{|c|c|}
\hline $\begin{array}{l}\text { Inflammatory } \\
\text { demyelinating CNS* } \\
\text { disease }\end{array}$ & $\begin{array}{l}\text { - Acute disseminated encephalomyelitis } \\
\text { - Multiple sclerosis } \\
\text { - Neuromyelitis optica (Devic's disease) }\end{array}$ \\
\hline Cerebrovascular disease & $\begin{array}{l}\text { - Cerebral autosomal dominant } \\
\text { Arteriopathy with subcortical infarcts } \\
\text { and Leukoencephalopathy (CADASIL) } \\
\text { - Stroke } \\
\text { - Transient ischaemic attack }\end{array}$ \\
\hline $\begin{array}{l}\text { Vasculitic, connective } \\
\text { tissue, and autoimmune } \\
\text { disease }\end{array}$ & $\begin{array}{l}\text { - Antiphospholipid antibody syndrome } \\
\text { - Behçet disease } \\
\text { - Churg-Strauss syndrome } \\
\text { - Dermatomyositis } \\
\text { - Eales disease } \\
\text { - Limbic encephalitis } \\
\text { - Polyarteritis nodosa } \\
\text { - Primary CNS* vasculitis } \\
\text { - Sarcoidosis } \\
\text { - Sjögren syndrome } \\
\text { - Systemic lupus erythematosus } \\
\text { - Takayasu Disease } \\
\text { - Vogt-Koyanagi-Harada syndrome } \\
\text { - Wegener granulomatosis }\end{array}$ \\
\hline Infectious CNS $^{\star}$ disease & $\begin{array}{l}\text { - } \text { Creutzfeldt-Jakob disease } \\
\text { - Lyme disease } \\
\text { - Progressive multifocal } \\
\text { leukoencephalopathy } \\
\text { - Syphilis } \\
\text { - Toxoplasmosis } \\
\text { - Tuberculosis } \\
\text { - Viral encephalitis }\end{array}$ \\
\hline Malignancy & $\begin{array}{l}\text { - } \text { CNS }^{*} \text { metastases } \\
\text { - Primary CNS* Iymphoma } \\
\text { - Paraneoplastic syndrome }\end{array}$ \\
\hline Otologic disease & $\begin{array}{l}\text { - Cogan syndrome } \\
\text { - Ménière's disease } \\
\text { - Sudden sensorineural hearing loss }\end{array}$ \\
\hline Other & $\begin{array}{l}\text { - } \text { Cryoglobulinemia } \\
\text { - Lactate acidosis and stroke-like } \\
\text { - } \text { Episodes (MELAS) } \\
\text { - Marchiafava-Bignami disease } \\
\text { - Migraines } \\
\text { - Psychotic disorders }\end{array}$ \\
\hline
\end{tabular}

:Central nervous system (from Dörr et al., 2013 ${ }^{10}$, mod.). etry remains essential to confirm the presence of sensorineural hearing loss in patients presenting with concern for SS. While audiometric data on SS patients is limited, the observed sensorineural hearing loss appears to affect the low and middle frequencies, with an occasional loss appreciated at the high frequencies $4722-24$ 27. To date, Roeser and colleagues have provided the most comprehensive description of audiometric findings in SS patients. In their cases series of 23 patients, representing a total of 34 affected ears, most frequencies were found to be affected, with losses noted at 500 and $1,000 \mathrm{~Hz}$ and an overall "upsloping" pattern of sensorineural hearing loss (Fig. 2). Tympanometry for all 23 patients was found to be type A, indicating a normal middle ear system. The pure-tone average of the 34 affected ears was $41.5 \mathrm{~dB}$, with a mean percent binaural hearing loss of $26.4 \%$. The American Academy of Otolaryngology-Head and Neck Surgery hearing classification of this cohort demonstrated primarily type A (47\%) hearing, with losses also noted in categories type B (23.5\%), type C (6.0\%), and type D $(23.5 \%)$. Finally, word recognition scores were poor in this study group, with only $26.5 \%$ of affected ears exhibiting $100 \%$-word recognition ${ }^{24}$.

\section{b. Vestibular-evoked myogenic potentials}

Vestibular-evoked myogenic potentials can be applied in the workup of a patient with suspected SS, particularly if there is evidence of acute vestibular dysfunction upon clinical presentation. In a study by Magliulo and colleagues, they were able to successfully characterise a vestibular-evoked myogenic potential deficit, with both delayed latency and reduced amplitude in a patient with SS (Fig. 3). It is reasonable to extrapolate that the initial otologic damage in SS may involve a restricted portion of the vestibular apparatus ${ }^{32}$. Further investigation is necessary to explore such mechanistic details.

c. Laboratory investigations

Due to variability in presenting features of SS, individuals are often subjected to a myriad of diagnostic laboratory tests, often with few abnormal findings. Focused serologic testing is recommended, with expansion as indicated to evaluate for related differential diagnoses (Table II).

- Antibody markers. Mild elevation of antinuclear, antiphospholipid, anti-thyroid microsomal, anti-thyroid peroxidase, perinuclear anti-neutrophil cytoplasmic, and rheumatoid factor autoantibodies have all been reported in SS patients. In a case series by Jarius and colleagues of 25 SS patients, 3 patients (13.0\%) were reported to have anti-nuclear antibody positivity, 1 patient $(4.0 \%)$ was reported to have perinuclear antineutrophil cytoplasmic antibody positivity, 1 patient 
Table III. Susac syndrome classification.

\begin{tabular}{ll} 
Category & Definition \\
Suspected & One triad manifestation ${ }^{\dagger}$ \\
& One risk factor* \\
& 1. Female \\
& a. $20-40$ Years \\
& b. Within 1 year of pregnancy \\
& 2. Characteristic MRI findings \\
& a. Corpus callosum lesions \\
& b. Periventricular lesions \\
Incomplete & Two triad manifestations ${ }^{\dagger}$ \\
Complete & Three triad manifestations \\
\hline
\end{tabular}

: With no known risk factors for arteriosclerosis and/or coagulopathy. t: Clinical triad of encephalopathy, sensorineural hearing loss, and branch retinal artery occlusion. Susac syndrome classification system (from Vishnevskia-dai et al., $2016^{31}$, mod.).

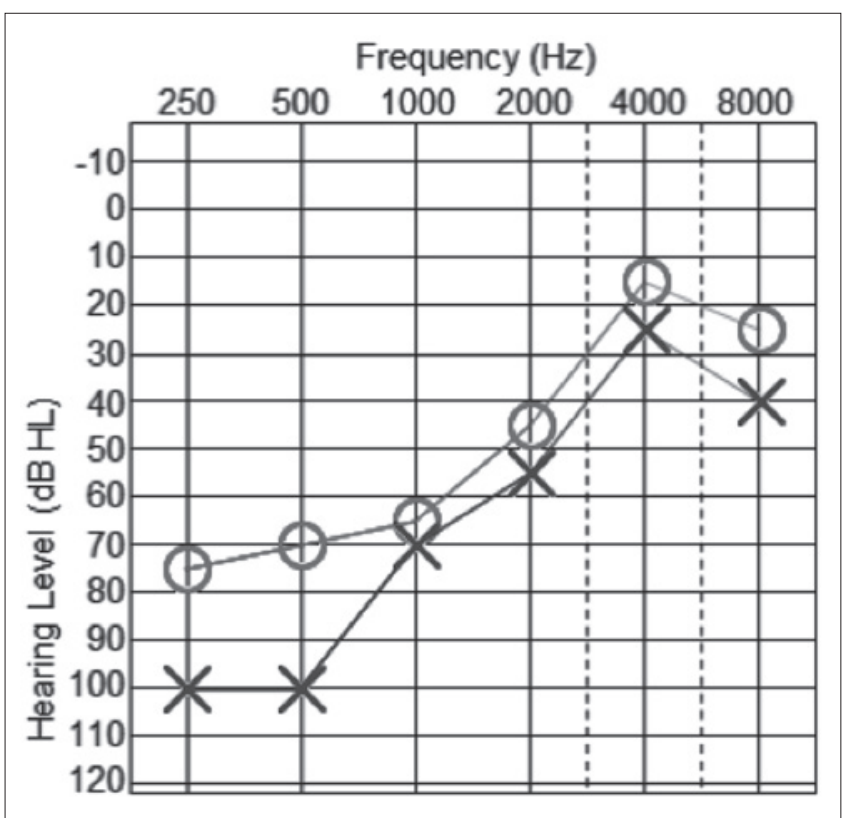

Fig. 2. 36-year-old female with sensorineural hearing loss in the setting of Susac Syndrome. Pure tone audiometry reveals bilateral asymmetric sensorineural hearing loss, predominantly at the low and middle frequencies. The right ear is graphed with a "o" and the left ear is graphed with an "x". These responses represent the air conduction results, masked if necessary in both ears. Speech recognition thresholds were 45 decibels in the right ear and 60 decibels in the left ear; word discrimination scores were $96 \%$ in the right ear and $88 \%$ in the left ear.

was reported to have anti-thyroid microsomal antibody positivity, and 1 patient (4.0\%) was reported to have anti-thyroid peroxidase antibody positivity ${ }^{33}$. Antiendothelial cell antibodies, a heterogeneous family of antibodies which bind to various endothelial cell antigens, have also been detected in the serum of SS patients; it has been postulated to serve as a biomarker of disease activity ${ }^{34} 35$. In the case series by Jarius and

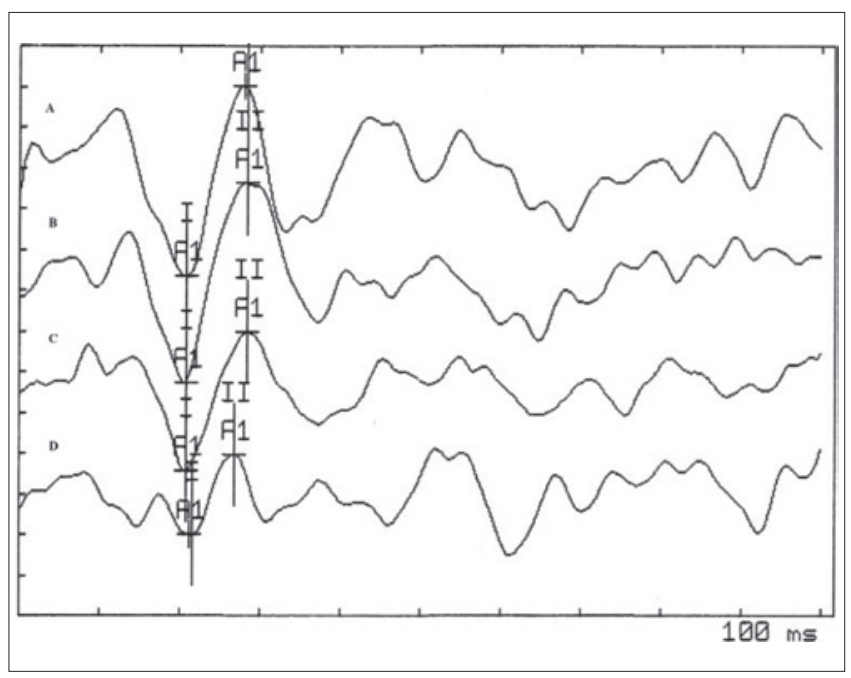

Fig 3. 24-year-old female with acute vertigo in the setting of Susac syndrome. Side, left; stimulus, logon; frequency, $500 \mathrm{~Hz}$; number of stimuli, 200; intensity, A 130, B 130, C 120, D 110 dB sound pressure level; masking, off; rate, 4/s; polarity, negative; sensitivity, $10 \mu \mathrm{V}$ per division; amplitude, $\mathrm{A} 23.42$, B 24.35, C 34.09, D 9.68; latency A 20.8, B 20.8, C 20.8, D 21.6 (from Magliulo et al., $2008{ }^{32}$, used with permission from Giuseppe Magliulo).

colleagues, 7 patients (28\%) were found to have antiendothelial cell antibody positivity ${ }^{33}$. However, it remains uncertain whether these antibodies are unique to SS or represent a non-specific epiphenomenon. At this time, it remains a non-specific assay as it has been reported in other vasculitic and autoimmune processes including systemic lupus erythematosus, rheumatoid arthritis, Sjögren syndrome and sarcoidosis. There are several methods to detect anti-endothelial cell antibodies but sensitivity and specificity of these antibodies as biomarkers for SS have yet to be established.

- Haematologic panel. A complete blood count with differential may be performed, which occasionally reveals monocytosis and eosinophilia ${ }^{36}$. Similarly, mild elevation in acute phase reactants including erythrocyte sedimentation rate and $\mathrm{C}$-reactive protein has been reported in SS patients. Elevated levels of factor VIII and von Willebrand factor antigen have also been documented in SS, likely due to endothelial perturbation, as the close association of these factors with endothelium would be consistent with the observed endotheliopathy ${ }^{19}$.

- Cerebrospinal fluid analysis. Cerebrospinal fluid examination reveals lymphocytic pleocytosis (5-30 cells/ $\mathrm{mm}^{3}$ ), elevated protein levels (range: $100-3000 \mathrm{mg} / \mathrm{dl}$ ), usually during the encephalopathic phase, and occasionally elevated myelin basic protein ${ }^{37}$. In a case series by Mateen and colleagues of 29 patients with SS, the mean cerebrospinal fluid protein level was found to be $130 \mathrm{mg} / \mathrm{dl}$ (median: 129, range: 35-268, normal range: 
$15-45 \mathrm{mg} / \mathrm{dl}$ ), with a mean cell count of 14 cells $/ \mathrm{ml}$ (median: 5, range: 0-86, normal range: $0-5$ cells $/ \mathrm{ml}$ ) ${ }^{38}$. Analysis for oligoclonal bands is often performed due to overlapping clinical features of SS with multiple sclerosis as SS patients have no evidence of oligoclonal bands or an elevated Immunoglobulin $\mathrm{G}$ index.

\section{d. Radiographic imaging}

Subclinical pathology occurs in many patients with suspected SS without overt clinical manifestations, prompting emphasis on an appropriate diagnostic workup with dedicated imaging to confirm the diagnosis of SS. To date, SS patients with documented evidence of sensorineural hearing loss lack visualised radiographic aberrations within the external, middle and inner ear ${ }^{24}$.

- Computed tomography. Computed tomography of the brain is typically normal in the initial stages of SS, however with disease progression, this could progress to radiographically evident cortical atrophy ${ }^{23}$. In parallel, singlephoton emission computed tomography of the brain can also be utilised which reveals multifocal involvement corresponding to areas of cerebral hypoperfusion, confirming the presence of microangiopathy seen in $\mathrm{SS}^{3}$.

- Magnetic resonance imaging. Magnetic resonance imaging is the neuroimaging study of choice to evaluate for SS. Typical findings include central corpus callosum involvement and brain infarctions (Fig. 4). The cerebellum, cerebellar peduncles, brain stem and thalamus are less frequently involved. The deep gray matter is involved in approximately $70 \%$ of SS cases and the leptomeninges is affected in about $33 \%$ of SS cases ${ }^{39}$. T2-weighted sequences typically reveal multifocal, small, hyperintense foci which mainly involve the central aspect of the corpus callosum with peripheral sparing. Fluid-attenuated inversion recovery sequences may also show lesions in the corpus callosum centrally located in the periventricular and subcortical white matter. T1-weighted sequences reveal hypointense areas during the subacute or late phase of $\mathrm{SS}^{340}$. During periods of encephalopathy, the corpus callosum is universally affected and reveals a characteristic pattern of small-to-large round white matter lesions ("snowballs") best visualised on sagittal T2-weighted fluid-attenuated inversion recovery sequences and linear defects ("spokes") found in the central fibres of the corpus callosum. As acute changes resolve over time, central callosal lesions develop ("holes") and are best visualised on sagittal T1-weighted sequences ${ }^{41}$. Diffusion tensor imaging can also be used to evaluate for SS as it is specifically sensitive to structural impairment of axonal integrity based on normal values for fractional anisotropy, a measure which reflects spatial directionality of water diffusion ${ }^{42}$. The mean fractional ani- sotropy reduction in the genu of the corpus callosum and prefrontal white matter, as well as widespread impairment of white matter fibre integrity and fiber disruption involving the genu of the corpus callosum, appears to be typical for SS, revealing microstructural degeneration of cerebral fibres and extensive microstructural dysfunction ${ }^{43} 44$.

\section{e. Temporal bone histopathology}

The first description of otopathologic findings in SS was described by Francis and colleagues in 2011 who harvested two post-mortem temporal bones from a 51-year-old female with SS and audiometrically confirmed bilateral severe sensorineural hearing loss without concomitant vestibular symptomology ${ }^{8}$. Pertinent histopathologic findings in SS are outlined in detail below with generalised atrophy and degeneration involving the apical half of the cochlear duct with preservation of cochlear neurons (Fig. 5).

- Cochlea. The organs of Corti were found to be absent or represented by a mound of supporting cells and devoid of hair cells. The tectorial membranes revealed marked pathologic changes, which were more extensive in the left ear and characterised by cellular encapsulation and detachment from the spiral limbus. The lateral cochlear walls revealed near-total atrophy of the stria vascularis and severe degeneration of spiral ligament fibrocytes with replacement via connective tissue cells. The stria vascularis revealed patchy atrophy in the 3-7 $\mathrm{mm}$ region on the right and

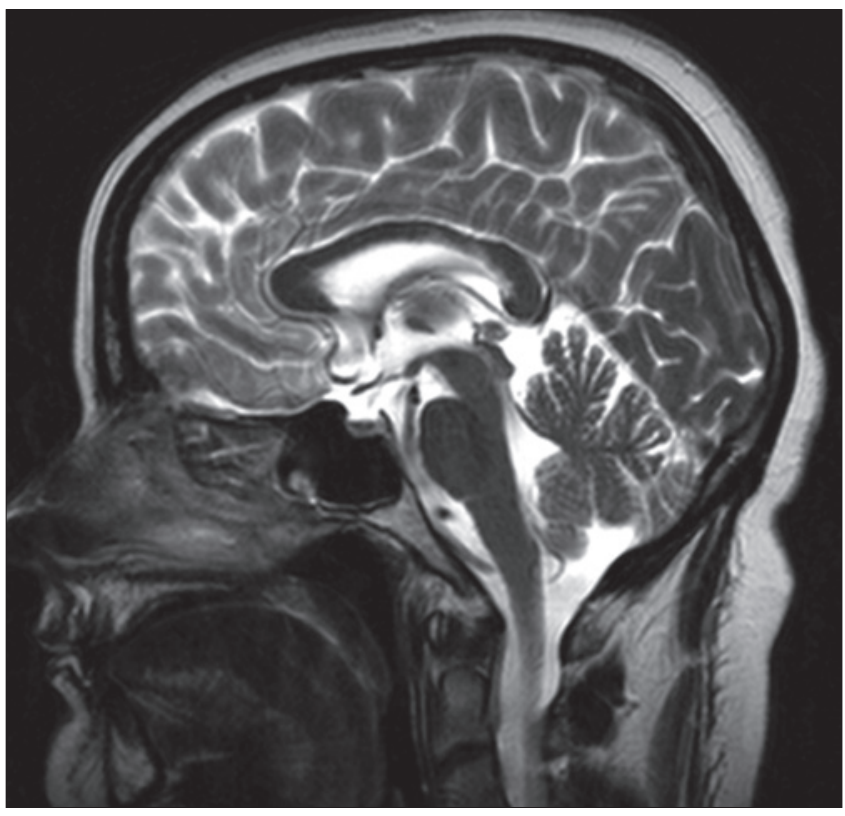

Fig. 4. 43-year-old female with Susac syndrome characterised by the clinical triad of encephalopathy, branch retinal artery occlusion, and sensorineural hearing loss. T2-weighted midline sagittal imaging reveals mild thinning of the corpus callosum near the junction of the body and the splenium. 
0-3 mm region in the left. Some capillaries within the stria vascularis revealed likely occlusion of the vessel lumen by an acellular substance or thickening of the vessel wall. The number of capillaries appeared to be reduced in the lateral cochlear wall of the middle and apical turns. The scala media in the middle and apical turns demonstrated scattered cellular debris, likely secondary to sloughing cells degenerating within the stria vascularis and organ of Corti. Finally, dendrites leading up to the organ of Corti similarly revealed atrophy in the middle and apical turns ${ }^{8}$.

- Saccule. The saccular macula in the left ear reveals diminution of type I hair cells; the saccular lumen in both ears contains free-floating cells, arranged in strands and folded strips. The origin of these cells is unclear, but they could represent detached cells which formerly lined the saccular duct ${ }^{8}$.

- Leptomeninges. The leptomeninges within the internal auditory canals revealed numerous psammoma bodies. Several small arterioles within the leptomeninges also revealed thickening of the vessel lumen to the point of occlusion. In some instances, there was cellular proliferation within or around the vessel wall causing occlusion, whereas other areas revealed a dense basophilic material occluding the vessel lumen ${ }^{8}$.

\section{Management principles}

Given the small number of SS cases reported worldwide as well as an incomplete understanding of disease pathogenesis, determination of an optimal treatment strategy remains difficult. As the observed clinical fractures are postulated to be vasculitic in nature, management of SS is primarily focused on anti-inflammatory and disease-modifying therapies. Although recommendations for treatment protocols do exist, it is important to note most therapies are based on limited cohorts of SS patients. As such, response to proposed medical regimens may be highly variable based upon individual disease characteristics. Initial treatment protocols were first designed for patients presenting with primarily encephalopathic forms of SS; however, current literature supports a different approach for patients with the recurrent branch retinal artery occlusion and hearing loss subset of $\mathrm{SS}^{34}$. This has prompted in parallel a significant interest in the exploration of otologic surgical interventions for SS patients ${ }^{525}$. Regardless of disease subtype, it is increasingly evident as well as imperative that early, aggressive treatment should be initiated whenever possible ${ }^{1534546}$.

\section{a. Corticosteroids}

In the acute phase, intravenous steroids are employed in an aggressive fashion as the initial treatment modality. Treat-

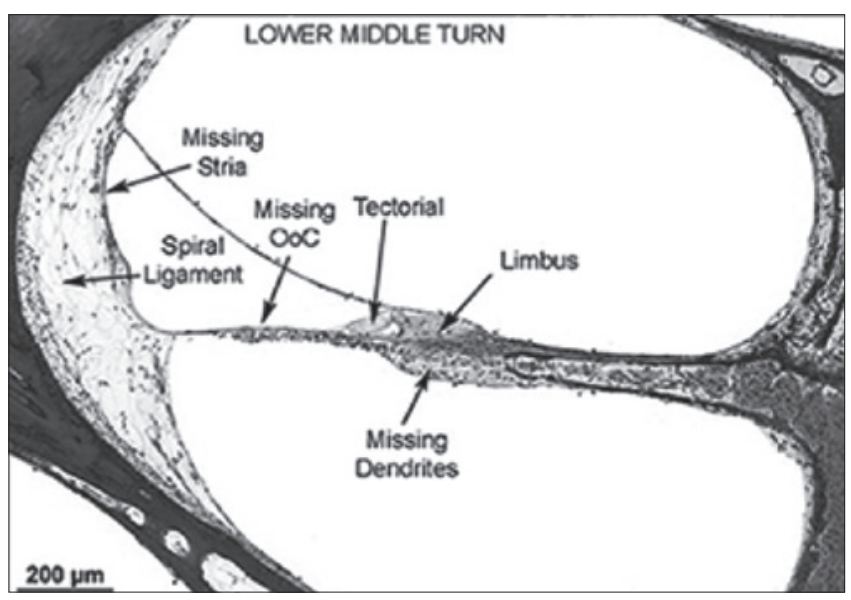

Fig. 5. 51-year old female with Susac syndrome and postmortem histopathological findings of the right temporal bone. Used with permission from Chadi Makary. Lower middle turn reveals widespread atrophy and degeneration. The organ of Corti is absent. The tectorial membrane is retracted and partially covered by cells. The spiral limbus reveals patchy atrophy and absent dendrites within the osseous spiral lamina. The stria vascularis is completely atrophic with significant loss of fibrocytes within the spiral ligament (from Francis et al., $2011^{8}$, mod.).

ment begins with intravenous methylprednisone $1,000 \mathrm{mg} /$ day over 3 days, followed by high dose oral prednisone 60 $80 \mathrm{mg} /$ day for a period of 2-4 weeks. This is followed by a slow taper with a daily dose reduction of $10 \%$ every 2 weeks, with a maintenance dose of 5-10 mg every other day ${ }^{45}$. An alternative to a prolonged course of oral prednisone, given long-term ramifications of corticosteroid use, is to utilize frequent pulses of intravenous methylprednisone, which may be beneficial particularly for SS relapses ${ }^{15}$. When inner ear disease accompanies central disease pathology, treatment of encephalopathic features using methylprednisone will simultaneously address any existing inner ear disease. Should otologic symptoms (acute sensorineural hearing loss, tinnitus and vertigo) predominate, it would be appropriate to promptly administer both intravenous methylprednisolone and intravenous immunoglobulin, with at least a moderate course of prednisone ${ }^{46}$.

b. Intravenous immunoglobulin and plasmapheresis Along with corticosteroids, concomitant intravenous immunoglobulin should be initiated in the first week of definitive treatment. The most commonly reported regimen consists of $2 \mathrm{~g} / \mathrm{kg}$ the first week, followed by $2 \mathrm{~g} / \mathrm{kg}$ every $2-4$ weeks over a treatment period of 6 months ${ }^{45}$. Plasmapheresis may also have a role for SS patients with severe or relapsing patterns of disease who have not responded to conventional medical therapies ${ }^{38}$.

c. Immunomodulators

Immunomodulatory drugs should be initiated early in the 
treatment of SS patients suffering from severe disease. This consists of intravenous cyclophosphamide $10-15 \mathrm{mg} /$ $\mathrm{kg}$ spaced 2 weeks apart, followed by additional doses if no change in symptomatology is seen. If notable improvement in symptoms does occur, patients should be switched to mycophenolate mofetil $1000-1500 \mathrm{mg}$ twice daily for maintenance therapy. Alternatives to this regimen include a combination of mycophenolate mofetil and tacrolimus. This should be administered as mycophenolate mofetil $500 \mathrm{mg}$ twice daily + tacrolimus $2 \mathrm{mg}$ BID via intravenous formulation as maintenance therapy. For patients with extremely severe disease who fail to respond to previously described therapies, rituximab may be of benefit. This should be administered with an initial dose of $1,000 \mathrm{mg}$, followed by the same dose 2 weeks later and subsequent doses at intervals of 4-6 months and 6-12 months. In rare instances, new additive ischaemic "hits" to the cochlea may occur, even during active treatment with corticosteroids, intravenous immunoglobulin, mycophenolate mofetil and rituximab. As the window of opportunity to protect the inner ear is short, replacement of mycophenolate mofetil, with cyclophosphamide and mycophenolate mofetil plus tacrolimus, may be indicated. Intravenous cyclophosphamide should be administered at a dose of $10-15 \mathrm{mg} / \mathrm{kg}$ every 2 weeks for a total of 2 doses. If improvement is unsatisfactory, one additional dose may be administered in 2 weeks, with future doses every 3 weeks for a total of 3 doses followed with a final round every 4 weeks with a total of $1-3$ doses ${ }^{46}$.

\section{d. Anticoagulants}

Owing to the microischaemic and prothrombotic state associated with SS, some authors advocate to administer aspirin $81 \mathrm{mg}$ on a daily basis as part of a maintenance medical therapy ${ }^{2}$. Observations of improvement in sensorineural hearing loss with a combination of aspirin and nimodipine have also been reported, likely secondary to an improved microvascular blood supply within the cochlea ${ }^{547}$. Other anticoagulants, such as clopidogrel, dipyridamole and fondaparinux, have been tested, although their efficacy in the management of SS has yet to be established ${ }^{33}$.

\section{e. Transtympanic dexamethasone}

Transtympanic dexamethasone injections can be utilised in patients with SS and concomitant findings of sensorineural hearing loss confirmed via audiometry ${ }^{24}$. Advantages of intratympanic perfusion include a higher concentration of medication delivered locally to the inner ear, ease of administration, the diseased ear is preferentially treated without adversely affecting the contralateral ear, and potential avoidance of systemic side effects ${ }^{48}$. Transtympanic injection of dexamethasone in the acute phase of sensorineural hearing loss in the setting of SS may pro- vide transient benefit with respect to halting progressive hearing loss and justify more aggressive systemic immunotherapy on grounds of potential reversibility.

\section{f. Cochlear implantation}

Should conservative medical therapies and transtympanic dexamethasone injections fail to result in notable clinical improvement, cochlear implantation is recommended for SS patients with irreversible otologic disease. Currently, cochlear implantation has been successfully employed in 5 patients who met surgical candidacy within the otolaryngology literature. Postoperatively, there was evidence of functional hearing restoration and notable improvement in communication abilities for all implanted SS patients $^{21} 24$ 49-51.

\section{Prognosis}

Early diagnosis and treatment of SS is hallmark to avoid devastating neurological, visual, and auditory sequelae. Overall, the prognosis can be significantly improved following prompt, aggressive, and sustained therapies, although poor outcomes often result from delayed, suboptimal management with premature, rapid tapering of treatment schedules ${ }^{18}$. Three major clinical courses have been described in SS: monocyclic, polycyclic and chronic continuous. In the monocyclic course, the disease remits spontaneously after 1-2 years without evidence of recurrence. The polycyclic course includes variable periods of remission which may persist for years. SS patients with branch retinal artery occlusion and sensorineural hearing loss are at increased risk for following a prolonged polycyclic course. The chronic continuous course demonstrates variations in severity of symptoms. Remission may occur in this group but there is no clear evidence of complete disease diminution. It remains difficult on initial presentation to identify which patients may regress spontaneously or progress with more fulminant symptomology ${ }^{52}$. In addition, relapses after decades of quiescence have also been described, further muddling long-term outcomes ${ }^{53}{ }^{54}$. Accordingly, this disease entity requires lifetime monitoring in conjunction with an ophthalmologist, otolaryngologist, neurologist and rheumatologist. Risk factors for relapse include pregnancy, as SS patients will often be tapered off treatment regimens to minimise risk of foetal toxicity ${ }^{52}$. Unlike other features of this condition, sensorineural hearing loss associated with SS is often irreversible and sometimes progressive in nature, despite early and aggressive treatment ${ }^{1955}$. Surgical interventions, chiefly transtympanic dexamethasone as well as cochlear implantation, are viable options to halt progression of otologic disease and augment serviceable hearing for those who meet surgical candidacy, respectively. 


\section{Conclusions}

Although modern advances have significantly extended our scope of knowledge regarding SS, this enigmatic disorder remains incompletely understood. As insights into the pathophysiology of SS continue to evolve, research endeavours remain broadly focused within several disciplines, which may provide evidence-based standardised diagnostic and treatment protocols for disease sufferers in the future. The current body of available evidence suggests SS is likely due to a selective endotheliopathy of the systemic microvasculature, which appears to vasculitic in origin. This distinction is critical, as the inner ear is not an immunologically privileged site; cochlear injury typically occurs via a robust response to foreign and self-antigens through both humoral and cell-mediated mechanisms. Thus, not only should SS be routinely considered as part of the differential diagnosis in patients presenting with multi-system disease and hearing loss, but also it should be considered as a unique vasculitic otologic entity-akin, yet pathologically dissimilar to traditional autoimmune otologic disease. Important otologic manifestations of SS include fluctuating sensorineural hearing loss, tinnitus and vertigo. Audiometric evaluation typically reveals an upsloping pattern of sensorineural hearing loss in the low to middle frequencies. Given the observed sensorineural hearing loss is often irreversible and progressive, surgical interventions including transtympanic cexamethasone and cochlear implantation are viable options to halt disease progression and augment serviceable hearing for those who meet device candidacy, respectively. Multicentre prospective studies are needed to expand our experience with SS and plan future consensus-based recommendations. This comprehensive review has been designed to serve as a centralised and up-to-date resource for clinicians and should be updated whenever novel and highlevel clinical evidence becomes available.

\section{Acknowledgements}

The authors report no funding sources/conflict of interest and would like to thank Kimberly Walker, Division of Outcomes Research and Quality, for formatting the manuscript.

\section{Conflict of interest statement}

None declared.

\section{References}

1 Susac JO, Hardman JM, Selhorst JB. Microangiopathy of the brain and retina. Neurology 1979;29:313-6.

2 Kleffner I, Duning T, Lohmann H, et al. A brief review of Susac syndrome. J Neurol Sci 2012;322:35-40.
3 García-Carrasco M, Jiménez-Hernández C, Jiménez-Hernández M, et al. Susac's syndrome: an update. Autoimmun Rev 2011;10:548-52.

4 Do TH, Fisch C, Evoy F. Susac syndrome: report of four cases and review of the literature. AJNR Am J Neuroradiol 2004;25:382-8.

5 Fox RJ, Costello F, Judkins AR, et al. Treatment of Susac syndrome with gamma globulin and corticosteroids. J Neurol Sci 2006;251:17-22.

6 O'Halloran HS, Pearson PA, Lee WB, et al. Microangiopathy of the brain, retina, and cochlea (Susac syndrome). A report of five cases and a review of the literature. Ophthalmology 1998;105:1038-44.

7 Petty GW, Engel AG, Younge BR, et al. Retinocochleocerebral vasculopathy. Medicine (Baltimore) 1998;77:12-40.

8 Francis HW, Makary C, Halpin C, et al. Temporal bone findings in a case of Susac's syndrome. Otol Neurotol 2011;32:1198-204.

9 Egan RA, Ha Nguyen T, Gass DM, et al. Retinal arterial wall plaques in Susac syndrome. Am J Ophthalmol 2003;135:483-6.

10 Dörr J, Krautwald S, Wildemann B, et al. Characteristics of Susac syndrome: a review of all reported cases. Nat Rev Neurol 2013;9:307-16.

11 Greco A, De Virgilio A, Gallo A, et al. Susac's syndrome pathogenesis, clinical variants and treatment approaches. Autoimmun Rev 2014;13:814-21.

12 Susac JO. Susac's syndrome: the triad of microangiopathy of the brain and retina with hearing loss in young women. Neurology 1994;44:591-3.

13 Aubert-Cohen F, Klein I, Alexandra JF, et al. Longterm outcome in Susac syndrome. Medicine 2007;86:93-102.

14 Magro CM. Susac's syndrome: an autoimmune endotheliopathy. Paper presented at $1^{\text {st }}$ Susac's Syndrome Symposium. Columbus, OH: Ohio State University; 2005.

15 Rennebohm RM, Susac JO. Treatment of Susac's syndrome. J Neurol Sci 2007;257:215-20.

16 Pawate S, Agarwal A, Moses H, et al. The spectrum of Susac's syndrome. Neurol Sci 2009;30:59-64.

17 Papo T, Biousse V, Lehoang P, et al. Susac syndrome. Medicine 1998;77:3-11.

18 Bitra RK, Eggenberger E. Review of Susac syndrome. Curr Opin Ophthalmol 2011;22:472-6.

19 Saw VPJ, Canty PA, Green CM, et al. Susac syndrome: Microangiopathy of the retina, cochlea and brain. Clin Exp Ophthalmol 2000;28:373-81.

20 Gass JDM. Stereoscopic atlas of macular disease: diagnosis and treatment. $4^{\text {th }}$ ed. St. Louis: Mosby; 1997.

21 Bittencourt AG, Santos AF, Goffi-gomez MV, et al. Microangiopathy of the inner ear, deafness, and cochlear implantation in a patient with Susac syndrome. Acta Otolaryngol 2011;131:1123-8.

22 Karelle S, Demanez L, Zangerle PF, et al. Sudden sensorineural hearing loss: when ophthalmology meets otolaryngology. B-Ent 2012;8:135-9.

23 Gross M, Banin E, Eliashar R, et al. Susac syndrome. Otol Neurotol 2004;25:470-3. 
24 Roeser MM, Driscoll CL, Shallop JK, et al. Susac syndrome - a report of cochlear implantation and review of otologic manifestations in twenty-three patients. Otol Neurotol 2009;30:34-40.

25 Yurtsever B, Çabalar M, Kaya $\mathrm{H}$, et al. A rare cause of hearing loss: Susac syndrome. J Int Adv Otol 2015;11:167-9.

26 Monteiro ML, Swanson RA, Coppeto JR, et al. A microangiopathic syndrome of encephalopathy, hearing loss and retinal arteriolar occlusions. Neurology 1985;35:1113-21.

27 Turner BW, Digre KB, Shelton C. Susac syndrome. Otolaryngol Head Neck Surg 1998;118:866-7.

28 Ishii T, Watanabe I, Suzuki J. Temporal bone findings in Cogan's syndrome. Acta Otolaryngol Suppl 1995;519:118-23.

29 Turc G, Monnet D, Dupin N, et al. Skin involvement in Susac's syndrome. J Neurol Sci 2011;305:152-5.

30 Dorr J, Jarius S, Wildemann B, et al. Susac syndrome: an interdisciplinary challenge. Nervenarzt 2011;82:1250-63.

31 Vishnevskia-dai V, Chapman J, Sheinfeld R, et al. Susac syndrome: clinical characteristics, clinical classification, and longterm prognosis. Medicine (Baltimore) 2016;95:e5223.

32 Magliulo G, Al-Ansi W, Parrotto D, et al. Susac syndrome and vestibular-evoked myogenic potentials. Otolaryngol Head Neck Surg 2008;138:542-3.

33 Jarius S, Neumayer B, Wandinger KP, et al. Anti-endothelial serum antibodies in a patient with Susac's syndrome. J Neurol Sci 2009;285:259-61.

34 Rennebohm R, Susac JO, Egan RA, et al. Susac's syndrome update. J Neurol Sci 2010;299:86-91.

35 Magro CM, Poe JC, Lubow M, et al. Susac syndrome: an organ-specific autoimmune endotheliopathy syndrome associated with anti-endothelial cell antibodies. Am J Clin Pathol 2011;136:903-12.

36 Westreich R, Chandrasekhar S. Is Susac syndrome (microangiopathy of the inner ear, retina, and central nervous system) an underdiagnosed cause of sensorineural hearing loss? Ear Nose Throat J 2008;87:E4-7.

37 Rennebohm RM, Lubow M, Rusin J, et al. Aggressive immunosuppressive treatment of Susac's syndrome in an adolescent: using treatment of dermatomyositis as a model. Pediatr Rheumatol Online J 2008;6:3.

38 Mateen FJ, Zubkov AY, Muralidharan R, et al. Susac syndrome: clinical characteristics and treatment in 29 new cases. Eur J Neurol 2012;19:800-11.
39 Raets I, Gelin G. Susac's syndrome: a clinical and radiological challenge. JBR-BTR 2012;95:355-6.

40 Allmendinger AM, Spektor V, Destian S. CT and MR imaging of Susac syndrome in a young male presenting with acute disorientation. Clin Imaging 2010;34:138-42.

41 Saenz R, Quan AW, Magalhaes A, et al. MRI of Susac's syndrome. AJR 2005;184:1688-90.

42 Deppe M, Duning T, Mohammadi S, et al. Diffusion-tensor imaging at $3 \mathrm{~T}$ : detection of white matter alterations in neurological patients on the basis of normal values. Invest Radiol 2007;42:338-45.

43 Kleffner I, Deppe M, Mohammadi S, et al. Neuroimaging in Susac's syndrome: focus on DTI. J Neurol Sci 2010;29:92-6.

44 Kleffner I, Deppe M, Mohammadi S, et al. Diffusion tensor imaging demonstrates fiber impairment in Susac syndrome. Neurology 2008;70:1867-9.

45 Rennebohm RM, Susac JO. Treatment of Susac's syndrome. Curr Treat Options Neurol 2008;10:67-74.

46 Rennebohm RM, Asdaghi N, Srivastava S, et al. Guidelines for treatment of Susac syndrome - an update. Int J Stroke 2018; 1747493017751737.

47 Manor RS, Ouaknine L, Ouaknine G. Susac-RED-M syndrome [abstract]. Neuroophthalmology 1994;14 (Suppl. 1):113.

48 Jackson LE, Silverstein H. Chemical perfusion of the inner ear. Otolaryngol Clin North Am 2002;35:639-53.

49 Lavinsky L, Scarton F, Lavinsky-Wolff M, et al. Successful cochlear implantation in a Susac syndrome patient. Braz J Otorhinolaryngol 2012;78:123.

50 Connell SS, Brodie HA. Role of cochlear implantation in Susac's syndrome. Otolaryngol Head Neck Surg 2004;131:P280.

51 Grover N, Whiteside OJ, Ramsden JD. Susac syndrome: outcome of unilateral cochlear implantation. J Laryngol Otol 2011;125:856-8.

52 Deane KD, Tyler KN, Johnson DW, et al. Susac syndrome and pregnancy: disease management. J Clin Rheumatol 2011;17:83-8.

53 Petty GW, Matteson EL, Younge BR, et al. Recurrence of Susac syndrome (retinocochleocerebral vasculopathy) after remission of 18 years. Mayo Clin Proc 2001;76:958-60.

54 Feresiadou A, Eriksson U, Larsen HC, et al. Recurrence of Susac syndrome following 23 years of remission. Case Rep Neurol 2014;6:171-5.

55 Bateman ND, Johnson IJ, Gibbin KP. Susac's syndrome: a rare cause of fluctuating sensorineural hearing loss. J Laryngol Otol 1997;111:1072-4.

Received: March 30, 2018 - Accepted: July 15, 2018

Address for correspondence: Huseyin Isildak, The Pennsylvania State University, College of Medicine, Department of Surgery, Division of Otolaryngology - Head and Neck Surgery, 500 University Drive, MC H091, Hershey, PA 17033-0850, USA. Tel. +1 717-531-8946. Fax +1 717-531-6160. E-mail: hisildak@pennstatehealth.psu.edu 